\title{
Dimensionality Reduction for Supervised Learning with Reproducing Kernel Hilbert Spaces
}

\author{
Kenji Fukumizu \\ Institute of Statistical Mathematics \\ 4-6-7 Minami-Azabu, Minato-ku, Tokyo 106-8569, Japan \\ Francis R. Bach \\ Computer Science Division \\ University of California \\ Berkeley, CA 94720, USA \\ Michael I. Jordan \\ Computer Science Division and Department of Statistics \\ University of California \\ Berkeley, CA 94720, USA
}

FUKUMIZU@ISM.AC.JP

FBACH@CS.BERKELEY.EDU

JORDAN@CS.BERKELEY.EDU

Editor: Chris Williams

\begin{abstract}
We propose a novel method of dimensionality reduction for supervised learning problems. Given a regression or classification problem in which we wish to predict a response variable $Y$ from an explanatory variable $X$, we treat the problem of dimensionality reduction as that of finding a low-dimensional "effective subspace" for $X$ which retains the statistical relationship between $X$ and $Y$. We show that this problem can be formulated in terms of conditional independence. To turn this formulation into an optimization problem we establish a general nonparametric characterization of conditional independence using covariance operators on reproducing kernel Hilbert spaces. This characterization allows us to derive a contrast function for estimation of the effective subspace. Unlike many conventional methods for dimensionality reduction in supervised learning, the proposed method requires neither assumptions on the marginal distribution of $X$, nor a parametric model of the conditional distribution of $Y$. We present experiments that compare the performance of the method with conventional methods.
\end{abstract}

Keywords: regression, dimensionality reduction, variable selection, feature selection, kernel methods, conditional independence.

\section{Introduction}

Many statistical learning problems involve some form of dimensionality reduction, either explicitly or implicitly. The goal may be one of feature selection, in which we aim to find linear or nonlinear combinations of the original set of variables, or one of variable selection, in which we wish to select a subset of variables from the original set. The setting may be unsupervised learning, in which a set of observations of a random vector $X$ are available, or supervised learning, in which desired responses or labels $Y$ are also available. Developing methods for dimensionality reduction requires being clear on the goal and the setting, as methods developed for one combination of goal and setting are not generally appropriate for 
another. There are additional motivations for dimensionality reduction that it is also helpful to specify, including: providing a simplified explanation of a phenomenon for a human (possibly as part of a visualization algorithm), suppressing noise so as to make a better prediction or decision, or reducing the computational burden. These various motivations are often complementary.

In this paper we study dimensionality reduction in the setting of supervised learning. Thus, we consider problems in which our data consist of observations of $(X, Y)$ pairs, where $X$ is an $m$-dimensional explanatory variable and where $Y$ is an $\ell$-dimensional response. The variable $Y$ may be either continuous or discrete. We refer to these problems generically as "regression" problems, which indicates our focus on the conditional probability density function $p_{Y \mid X}(y \mid x)$. In particular, our framework includes discriminative approaches to classification problems, where $Y$ is a discrete label.

We wish to solve a problem of feature selection in which the features are linear combinations of the components of $X$. In particular, our modeling framework posits that there is an $r$-dimensional subspace $S \subset \mathbb{R}^{m}$ such that

$$
p_{Y \mid X}(y \mid x)=p_{Y \mid \Pi_{S} X}\left(y \mid \Pi_{S} x\right),
$$

for all $x$ and $y$, where $\Pi_{S}$ is the orthogonal projection of $\mathbb{R}^{m}$ onto $S$. The subspace $S$ is called the effective subspace for regression. Based on a set of observations of $(X, Y)$ pairs, we wish to recover a matrix whose columns span the effective subspace. The effective subspace can help to provide explanation of the statistical relation between $X$ and $Y$, by isolating the feature vectors that capture that relation. Also, finding such a space can suppress noise, to the extent that the orthogonal direction to the effective subspace is noisy vis-a-vis the prediction of $Y$.

We approach the problem as a semiparametric statistical problem; in particular, we make no assumptions regarding the conditional distribution $p_{Y \mid \Pi_{S} X}\left(y \mid \Pi_{S} x\right)$, nor do we make any assumptions regarding the marginal distribution $p_{X}(x)$. That is, we wish to estimate a finite-dimensional parameter (a matrix whose columns span the effective subspace), while treating the distributions $p_{Y \mid \Pi_{S} X}\left(y \mid \Pi_{S} x\right)$ and $p_{X}(x)$ nonparametrically.

Having found an effective subspace, we may then proceed to build a parametric or nonparametric regression model on that subspace. Thus our approach is an explicit dimensionality reduction method for supervised learning that does not require any particular form of regression model, and can be used as a preprocessor for any supervised learner. This can be compared to the use of methods such as principal components analysis (PCA) in regression, which also make no assumption regarding the subsequent regression model, but fail to make use of the response variable $Y$.

There are a variety of related approaches in the literature, but most of them involve making specific assumptions regarding the conditional distribution $p_{Y \mid \Pi_{S} X}\left(y \mid \Pi_{S} x\right)$, the marginal distribution $p_{X}(x)$, or both. For example, classical two-layer neural networks involve a linear transformation in the first "layer," followed by a specific nonlinear function and a second layer (Bishop, 1995). Thus, neural networks can be seen as attempting to estimate an effective subspace based on specific assumptions about the regressor $p_{Y \mid \Pi_{S} X}(y \mid$ $\Pi_{S} x$ ). Similar comments apply to projection pursuit regression (Friedman and Stuetzle, 1981), ACE (Breiman and Friedman, 1985) and additive models (Hastie and Tibshirani, 
1986), all of which provide a methodology for dimensionality reduction in which an additive model $E[Y \mid X]=g_{1}\left(\beta_{1}^{T} X\right)+\cdots+g_{K}\left(\beta_{K}^{T} X\right)$ is assumed for the regressor.

Bayesian approaches to variable selection include the Automatic Relevance Determination (ARD) method proposed by Neal (1996) for neural networks. Vivarelli and Williams (1999) have adapted this approach to feature selection in the setting of Gaussian process regression. These methods also depend on a specific parametric model, on which a Bayesian prior distribution is assumed.

Canonical correlation analysis (CCA) and partial least squares (PLS, Höskuldsson, 1988, Helland, 1988) are classical multivariate statistical methods that can be used for dimensionality reduction in regression (Fung et al., 2002, Nguyen and Rocke, 2002). These methods are based on a linearity assumption for the regressor, however, and thus are quite strongly parametric.

The line of research that is closest to our work has its origin in a technique known as sliced inverse regression (SIR, Li, 1991). SIR is a semiparametric method for finding effective subspaces in regression. The basic idea is that the range of the response variable $Y$ is partitioned into a set of "slices," and the sample means of the observations $X$ are computed within each slice. This can be viewed as a rough approximation to the inverse regression of $X$ on $Y$. For univariate $Y$ the method is particularly easy to implement. Noting that the inverse regression must lie in the effective subspace if the forward regression lies in such a subspace, principal component analysis is then used on the sample means to find the effective subspace. Li (1991) has shown that this approach can find effective subspaces, but only under strong assumptions on the marginal distribution $p_{X}(x)$ - in particular, the marginal distribution must be elliptically symmetric.

Further developments in the wake of SIR include principal Hessian directions (pHd, Li, 1992), and sliced average variance estimation (SAVE, Cook and Weisberg, 1991, Cook and Yin, 2001). These are all semiparametric methods in that they make no assumptions about the regressor (see also Cook, 1998). However, they again place strong restrictions on the probability distribution of the explanatory variables. If these assumptions do not hold, there is no guarantee of finding the effective subspace.

There are also related nonparametric approaches that estimate the derivative of the regressor to achieve dimensionality reduction, based on the fact that the derivative of the conditional expectation $E\left[y \mid B^{T} x\right]$ with respect to $x$ belongs to the effective subspace (Samarov, 1993, Hristache et al., 2001). However, nonparametric estimation of derivatives is quite challenging in high-dimensional spaces.

There are also dimensionality reduction methods with a semiparametric flavor in the area of classification, notably the work of Torkkola (2003), who has proposed using nonparametric estimation of the mutual information between $X$ and $Y$, and subsequent maximization of this estimate of mutual information with respect to a matrix representing the effective subspace.

In this paper we present a novel semiparametric method for dimensionality reduction that we refer to as Kernel Dimensionality Reduction (KDR). KDR is based on the estimation and optimization of a particular class of operators on reproducing kernel Hilbert spaces (Aronszajn, 1950). Although our use of reproducing kernel Hilbert spaces is related to their role in algorithms such as the support vector machine and kernel PCA (Boser et al., 1992, Vapnik et al., 1997, Schölkopf et al., 1998), where the kernel function allows linear op- 
erations in function spaces to be performed in a computationally-efficient manner, our work differs in that it cannot be viewed as a "kernelization" of an underlying linear algorithm. Rather, we use reproducing kernel Hilbert spaces to provide characterizations of general notions of independence, and we use these characterizations to design objective functions to be optimized. We build on earlier work by Bach and Jordan (2002), who showed how to use reproducing kernel Hilbert spaces to characterize marginal independence between pairs of variables, and thereby design an objective function for independent component analysis. In the current paper, we extend this line of work, showing how to characterize conditional independence using reproducing kernel Hilbert spaces. We achieve this by expressing conditional independence in terms of covariance operators on reproducing kernel Hilbert spaces.

How does conditional independence relate to our dimensionality reduction problem? Recall that our problem is to find a projection $\Pi_{S}$ of $X$ onto a subspace $S$ such that the conditional probability of $Y$ given $X$ is equal to the conditional probability of $Y$ given $\Pi_{S} X$. This is equivalent to finding a projection $\Pi_{S}$ which makes $Y$ and $\left(I-\Pi_{S}\right) X$ conditionally independent given $\Pi_{S} X$. Thus we can turn the dimensionality reduction problem into an optimization problem by expressing it in terms of covariance operators.

In a presence of a finite sample, we need to estimate the covariance operator so as to obtain a sample-based objective function that we can optimize. We derive a natural plugin estimate of the covariance operator, and find that the resulting estimate is identical to the kernel generalized variance that has been described earlier by Bach and Jordan (2002) in the setting of independent component analysis. In that setting, the goal is to measure departures from independence, and the minimization of the kernel generalized variance can be viewed as a surrogate for minimizing a certain mutual information. In the dimensionality reduction setting, on the other hand, the goal is to measure conditional independence, and minimizing the kernel generalized variance can be viewed as a surrogate for maximizing a certain mutual information. Not surprisingly, the derivation that leads to the kernel generalized variance that we present here is quite different from the one presented in the earlier work on kernel ICA. Moreover, the argument that we present here can be viewed as providing a rigorous foundation for other, more heuristic, ways in which the kernel generalized variance has been used, including the model selection algorithms for graphical models presented by Bach and Jordan (2003b).

The paper is organized as follows. In Section 2, we introduce the problem of dimensionality reduction for supervised learning, and describe its relation with conditional independence and mutual information. Section 3 derives the objective function for estimation of the effective subspace for regression, and describes the KDR method. All of the mathematical details needed for the results in Section 3 are presented in the Appendix, which also provides a general introduction to covariance operators in reproducing kernel Hilbert spaces. In Section 4, we present a series of experiments that test the effectiveness of our method, comparing it with several conventional methods. Section 5 describes an extension of KDR to the problem of variable selection. Section 6 presents our conclusions. 


\section{Dimensionality Reduction for Regression}

We consider a regression problem, in which $Y$ is an $\ell$-dimensional random vector, and $X$ is an $m$-dimensional explanatory variable. (Note again that we use "regression" in a generic sense that includes both continuous and discrete $Y$ ). The probability density function of $Y$ given $X$ is denoted by $p_{Y \mid X}(y \mid x)$. Assume that there is an $r$-dimensional subspace $S \subset \mathbb{R}^{m}$ such that

$$
p_{Y \mid X}(y \mid x)=p_{Y \mid \Pi_{S} X}\left(y \mid \Pi_{S} x\right),
$$

for all $x$ and $y$, where $\Pi_{S}$ is the orthogonal projection of $\mathbb{R}^{m}$ onto $S$. The subspace $S$ is called the effective subspace for regression.

The problem that we treat here is that of finding the subspace $S$ given an i.i.d. sample $\left\{\left(X_{1}, Y_{1}\right), \ldots,\left(X_{n}, Y_{n}\right)\right\}$ from $p_{X}$ and $p_{Y \mid X}$. The crux of the problem is that we assume no $a$ priori knowledge of the regressor, and place no assumptions on the conditional probability $p_{Y \mid X}$.

As in the simpler setting of principal component analysis, we make the (generally unrealistic) assumption that the dimensionality $r$ is known and fixed. While choosing the dimensionality is a very important problem, we separate it from the task of finding the best subspace of a fixed dimensionality, and mainly focus on the latter problem. In Section 6, we briefly discuss various approaches to the estimation of the dimensionality.

The notion of effective subspace can be formulated in terms of conditional independence. Let $(B, C)$ be the $m$-dimensional orthogonal matrix such that the column vectors of $B$ span the subspace $S$, and define $U=B^{T} X$ and $V=C^{T} X$. Because $(B, C)$ is an orthogonal matrix, we have

$$
p_{X}(x)=p_{U, V}(u, v), \quad p_{X, Y}(x, y)=p_{U, V, Y}(u, v, y),
$$

for the probability density functions. From Equation (2), Equation (1) is equivalent to

$$
p_{Y \mid U, V}(y \mid u, v)=p_{Y \mid U}(y \mid u) .
$$

This shows that the effective subspace $S$ is the one which makes $Y$ and $V$ conditionally independent given $U$ (see Figure 1).

Mutual information provides another point of view on the equivalence between conditional independence and the existence of the effective subspace. From Equation (2), it is straightforward to see that

$$
I(Y, X)=I(Y, U)+E_{U}[I(Y|U, V| U)]
$$

where $I(Z, W)$ denotes the mutual information defined by

$$
I(Z, W):=\iint p_{Z, W}(z, w) \log \frac{p_{Z, W}(z, w)}{p_{Z}(z) p_{W}(w)} d z d w .
$$

Because Equation (1) means $I(Y, X)=I(Y, U)$, the effective subspace $S$ is characterized as the subspace which retains the mutual information of $X$ and $Y$ by the projection onto that subspace, or equivalently, which gives $I(Y|U, V| U)=0$. This is again the conditional independence of $Y$ and $V$ given $U$. 


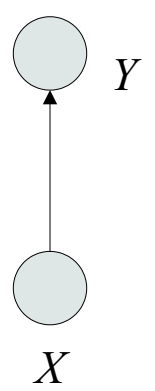

(a)

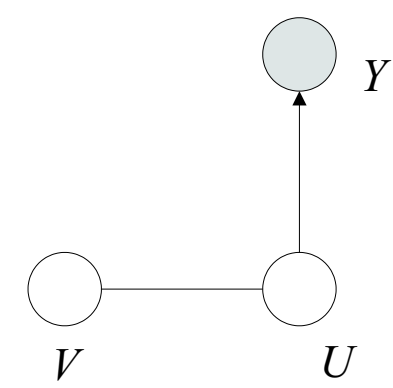

(b)

Figure 1: Graphical representation of dimensionality reduction for regression. The variables $Y$ and $V$ are conditionally independent given $U$, where $X=(U, V)$.

The expression in Equation (3) can be understood in terms of the decomposition of the mutual information according to a tree-structured graphical model - a quantity that has been termed the T-mutual information by Bach and Jordan (2003a). Considering the tree $Y-U-V$ in Figure 1(b), we have that the T-mutual information $I^{T}$ is given by

$$
I^{T}=I(Y, U, V)-I(Y, U)-I(U, V) .
$$

This is equal to the KL-divergence between a probability distribution on $(Y, U, V)$ and its projection onto the family of distributions that factor according to the tree; that is, the set of distributions that verify $Y \Perp V \mid U$. Using Equation (2), we can easily see that $I(Y, U, V)=I(Y, X)+I(U, V)$, and thus we obtain

$$
I^{T}=I(Y, X)-I(Y, U)=E_{U}[I(Y|U, V| U)]
$$

Then, dimensionality reduction for regression can be viewed as the problem of minimizing the T-mutual information for the fixed tree structure in Figure 1(b).

From Equation (3) and Equation (4), we see that there are two approaches to solve the problem of dimensionality reduction for regression; one is to maximize the mutual information $I(Y, U)$, and the other is to make $Y$ and $V$ conditionally independent given $U$. We choose the latter in deriving our method. Direct evaluation of mutual information is not a straightforward task in general, because it requires an explicit form for the probability density functions. Although assuming a specific probability model leads readily to a solution, it implies a restriction of the range of problems to which the method can be applied, and does not satisfy our goal of developing a general semiparametric method. Nonparametric estimation of the probabilities also provides an approach to evaluation of mutual information. However, nonparametric estimation and numerical integration do not give accurate estimates for high-dimensional variables, and it is a challenge to make a nonparametric method viable (Torkkola, 2003). An alternative semiparametric approach was presented by Bach and Jordan (2002), who showed that the kernel generalized variance could serve as a surrogate for the mutual information in the setting of independent component analysis. 
Our approach to dimensionality reduction also makes use of the kernel generalized variance. However, we do not take the mutual information as our point of departure, because we expect to be far from the setting of mutual independence in which Bach and Jordan (2002) showed that the mutual information and kernel generalized variance are closely related. Instead, we take an entirely different path, presenting a rigorous characterization of conditional independence using reproducing kernels, and showing that this characterization leads once again to the kernel generalized variance.

\section{Kernel Method for Dimensionality Reduction in Regression}

In this section we present our kernel-based method for dimensionality reduction. We discuss the basic definition and properties of cross-covariance operators on reproducing kernel Hilbert spaces, derive an objective function for characterizing conditional independence using cross-covariance operators, and finally present a sample-based objective function based on this characterization.

\subsection{Cross-Covariance Operators on Reproducing Kernel Hilbert Spaces}

We use cross-covariance operators on reproducing kernel Hilbert spaces to derive an objective function for dimensionality reduction. While cross-covariance operators are generally defined for random variables in Banach spaces (Vakhania et al., 1987, Baker, 1973), the theory is much simpler for reproducing kernel Hilbert spaces. We summarize only basic mathematical facts in this subsection, and defer the details to the Appendix. Let $(\mathcal{H}, k)$ be a reproducing kernel Hilbert space of functions on a set $\Omega$ with a positive definite kernel $k: \Omega \times \Omega \rightarrow \mathbb{R}$. The inner product of $\mathcal{H}$ is denoted by $\langle\cdot, \cdot\rangle_{\mathcal{H}}$. We consider only real Hilbert spaces for simplicity. The most important aspect of reproducing kernel Hilbert spaces is the reproducing property:

$$
\langle f, k(\cdot, x)\rangle_{\mathcal{H}}=f(x) \quad \text { for all } x \in \Omega \text { and } f \in \mathcal{H} .
$$

Throughout this paper we use the Gaussian kernel

$$
k\left(x_{1}, x_{2}\right)=\exp \left(-\left\|x_{1}-x_{2}\right\|^{2} / \sigma^{2}\right)
$$

which corresponds to a Hilbert space of smooth functions.

Let $\left(\mathcal{H}_{1}, k_{1}\right)$ and $\left(\mathcal{H}_{2}, k_{2}\right)$ be reproducing kernel Hilbert spaces over measurable spaces $\left(\Omega_{1}, \mathcal{B}_{1}\right)$ and $\left(\Omega_{2}, \mathcal{B}_{2}\right)$, respectively, with $k_{1}$ and $k_{2}$ measurable. For a random vector $(X, Y)$ on $\Omega_{1} \times \Omega_{2}$, the cross-covariance operator from $\mathcal{H}_{1}$ to $\mathcal{H}_{2}$ is defined by the relation

$$
\left\langle g, \Sigma_{Y X} f\right\rangle_{\mathcal{H}_{2}}=E_{X Y}[f(X) g(Y)]-E_{X}[f(X)] E_{Y}[g(Y)]
$$

for all $f \in \mathcal{H}_{1}$ and $g \in \mathcal{H}_{2}$. Equation (5) implies that the covariance of $f(X)$ and $g(Y)$ is given by the action of the linear operator $\Sigma_{Y X}$ and the inner product. (See the Appendix for a basic exposition of cross-covariance operators.)

Covariance operators provide a useful framework for discussing conditional probability and conditional independence. As we show in Corollary 3 of the Appendix, the following 
relation holds between the conditional expectation and the cross-covariance operator, given that $\Sigma_{X X}$ is invertible: ${ }^{1}$

$$
E_{Y \mid X}[g(Y) \mid X]=\Sigma_{X X}^{-1} \Sigma_{X Y} g \quad \text { for all } g \in \mathcal{H}_{2}
$$

Equation (6) can be understood by analogy to the conditional expectation of Gaussian random variables. If $X$ and $Y$ are Gaussian random variables, it is well known that the conditional expectation is given by

$$
E_{Y \mid X}\left[a^{T} Y \mid X=x\right]=x^{T} \Sigma_{X X}^{-1} \Sigma_{X Y} a,
$$

for an arbitrary vector $a$, where $\Sigma_{X X}$ and $\Sigma_{X Y}$ are the variance-covariance matrices in the ordinary sense.

\subsection{Conditional Covariance Operators and Conditional Independence}

We derive an objective function for characterizing conditional independence using crosscovariance operators. Suppose we have random variables $X$ and $Y$ on $\mathbb{R}^{m}$ and $\mathbb{R}^{\ell}$, respectively. The variable $X$ is decomposed into $U \in \mathbb{R}^{r}$ and $V \in \mathbb{R}^{m-r}$ so that $X=(U, V)$. For the function spaces corresponding to $Y, U$ and $V$, we consider the reproducing kernel Hilbert spaces $\left(\mathcal{H}_{1}, k_{1}\right),\left(\mathcal{H}_{2}, k_{2}\right)$, and $\left(\mathcal{H}_{3}, k_{3}\right)$ on $\mathbb{R}^{\ell}, \mathbb{R}^{r}$, and $\mathbb{R}^{m-r}$, respectively, each endowed with Gaussian kernels. We define the conditional covariance operator $\Sigma_{Y Y \mid U}$ on $\mathcal{H}_{1}$ by

$$
\Sigma_{Y Y \mid U}:=\Sigma_{Y Y}-\Sigma_{Y U} \Sigma_{U U}^{-1} \Sigma_{U Y}
$$

where $\Sigma_{Y Y}, \Sigma_{U U}, \Sigma_{Y U}$ are the corresponding covariance operators. As shown by Proposition 5 in the Appendix, the operator $\Sigma_{Y Y \mid U}$ captures the conditional variance of a random variable in the following way:

$$
\left\langle g, \Sigma_{Y Y \mid U} g\right\rangle_{\mathcal{H}_{1}}=E_{U}\left[\operatorname{Var}_{Y \mid U}[g(Y) \mid U]\right]
$$

where $g$ is an arbitrary function in $\mathcal{H}_{1}$. As in the case of Equation (7), we can make an analogy to Gaussian variables. In particular, Equations (8) and (9) can be viewed as the analogs of the following well-known equality for the conditional variance of Gaussian variables:

$$
\operatorname{Var}\left[a^{T} Y \mid U\right]=a^{T}\left(\Sigma_{Y Y}-\Sigma_{Y U} \Sigma_{U U}^{-1} \Sigma_{U Y}\right) a
$$

It is natural to use minimization of $\Sigma_{Y Y \mid U}$ as the basis of a method for finding the most informative direction $U$. This intuition is justified theoretically by Theorem 7 in the Appendix. That theorem shows that

$$
\Sigma_{Y Y \mid U} \geq \Sigma_{Y Y \mid X} \quad \text { for any } \quad U
$$

and

$$
\Sigma_{Y Y \mid U}-\Sigma_{Y Y \mid X}=0 \quad \Longleftrightarrow \quad Y \Perp V \mid U,
$$

1. Even if $\Sigma_{X X}$ is not invertible, a similar fact holds. See Corollary 3. 
where, in Equation (10), the inequality should be understood as the partial order of selfadjoint operators. From these relations, the effective subspace $S$ can be characterized in terms of the solution to the following minimization problem:

$$
\min _{S} \Sigma_{Y Y \mid U}, \quad \text { subject to } U=\Pi_{S} X \text {. }
$$

In the following section we show how to turn this population-based criterion into a samplebased criterion that can be optimized in the presence of a finite sample.

\subsection{Kernel Generalized Variance for Dimensionality Reduction}

To derive a sample-based objective function from Equation (11), we have to estimate the conditional covariance operator from data, and choose a specific way to evaluate the size of self-adjoint operators. While there are many possibilities for approaching these two problems, we make a specific choice, adopting an approach which has been used successfully for independent component analysis (Bach and Jordan, 2002).

We describe a regularized empirical estimate of the cross-covariance operator. Define $\tilde{k}_{1}^{(i)} \in \mathcal{H}_{1}$ by $\tilde{k}_{1}^{(i)}=k_{1}\left(\cdot, Y_{i}\right)-\frac{1}{n} \sum_{j=1}^{n} k_{1}\left(\cdot, Y_{j}\right)$, and $\tilde{k}_{2}^{(i)} \in \mathcal{H}_{2}$ similarly using $U_{i}$. By replacing the expectation with the empirical average, the covariance is estimated by

$$
\left\langle f, \Sigma_{Y U} g\right\rangle_{\mathcal{H}_{1}} \approx \frac{1}{n} \sum_{i=1}^{n}\left\langle\tilde{k}_{1}^{(i)}, f\right\rangle\left\langle\tilde{k}_{2}^{(i)}, g\right\rangle .
$$

Let $\hat{K}_{Y}$ be the centralized Gram matrix (Bach and Jordan, 2002, Schölkopf et al., 1998), defined by

$$
\hat{K}_{Y}=\left(I_{n}-\frac{1}{n} \mathbf{1}_{n} \mathbf{1}_{n}^{T}\right) G_{Y}\left(I_{n}-\frac{1}{n} \mathbf{1}_{n} \mathbf{1}_{n}^{T}\right),
$$

where $\left(G_{Y}\right)_{i j}=k_{1}\left(Y_{i}, Y_{j}\right)$ is the Gram matrix and $\mathbf{1}_{n}=(1, \ldots, 1)^{T}$ is the vector with all elements equal to 1 . The matrix $\hat{K}_{U}$ is defined similarly using $\left\{U_{i}\right\}_{i=1}^{n}$. Then, it is easy to see that $\left\langle\tilde{k}_{1}^{(i)}, \tilde{k}_{1}^{(j)}\right\rangle=\left(\hat{K}_{Y}\right)_{i j}$ and $\left\langle\tilde{k}_{2}^{(i)}, \tilde{k}_{2}^{(j)}\right\rangle=\left(\hat{K}_{U}\right)_{i j}$. If we decompose $f$ and $g$ as $f=\sum_{\ell=1}^{n} a_{i} \tilde{k}_{1}^{(\ell)}+f^{\perp}$ and $g=\sum_{m=1}^{n} b_{m} \tilde{k}_{2}^{(m)}+g^{\perp}$, where $f^{\perp}$ and $g^{\perp}$ are orthogonal to the linear hull of $\left\{\tilde{k}_{1}^{(i)}\right\}_{i=1}^{n}$ and $\left\{\tilde{k}_{2}^{(i)}\right\}_{i=1}^{n}$, respectively, we see that the covariance is approximated by

$$
\left\langle f, \Sigma_{Y U} g\right\rangle_{\mathcal{H}_{1}} \approx \sum_{\ell=1}^{n} \sum_{m=1}^{n} a_{\ell} b_{m}\left(\hat{K}_{Y} \hat{K}_{U}\right)_{l m} .
$$

Thus, by restricting the covariance operator $\Sigma_{Y U}$ to the $n$-dimensional subspaces spanned by $\left\{k_{1}^{(i)}\right\}_{i=1}^{n}$ and $\left\{k_{2}^{(i)}\right\}_{i=1}^{n}$, we can estimate the operator by

$$
\hat{\Sigma}_{Y U}=\hat{K}_{Y} \hat{K}_{U}
$$

In estimating $\Sigma_{Y Y}$ and $\Sigma_{U U}$, we use the same regularization technique as Bach and Jordan (2002). The empirical conditional covariance matrix $\hat{\Sigma}_{Y Y \mid U}$ is then defined by

$$
\hat{\Sigma}_{Y Y \mid U}:=\hat{\Sigma}_{Y Y}-\hat{\Sigma}_{Y U} \hat{\Sigma}_{U U}^{-1} \hat{\Sigma}_{U Y}=\left(\hat{K}_{Y}+\varepsilon I_{n}\right)^{2}-\hat{K}_{Y} \hat{K}_{U}\left(\hat{K}_{U}+\varepsilon I_{n}\right)^{-2} \hat{K}_{U} \hat{K}_{Y},
$$

where $\varepsilon>0$ is a regularization constant. 
The size of $\hat{\Sigma}_{Y Y \mid U}$ in the ordered set of positive definite matrices can be evaluated by its determinant. Although there are other choices for measuring the size of $\hat{\Sigma}_{Y Y \mid U}$, such as the trace and the largest eigenvalue, we focus on the determinant in this paper. Using the Schur decomposition $\operatorname{det}\left(A-B C^{-1} B^{T}\right)=\operatorname{det}\left(\begin{array}{cc}A & B \\ B^{T} & C\end{array}\right) / \operatorname{det} C$, the determinant of $\hat{\Sigma}_{Y Y \mid U}$ can be written as follows:

$$
\operatorname{det} \hat{\Sigma}_{Y Y \mid U}=\frac{\operatorname{det} \hat{\Sigma}_{[Y U][Y U]}}{\operatorname{det} \hat{\Sigma}_{U U}}
$$

where $\hat{\Sigma}_{[Y U][Y U]}$ is defined by

$$
\hat{\Sigma}_{[Y U][Y U]}=\left(\begin{array}{ll}
\hat{\Sigma}_{Y Y} & \hat{\Sigma}_{Y U} \\
\hat{\Sigma}_{U Y} & \hat{\Sigma}_{U U}
\end{array}\right)=\left(\begin{array}{cc}
\left(\hat{K}_{Y}+\varepsilon I_{n}\right)^{2} & \hat{K}_{Y} \hat{K}_{U} \\
\hat{K}_{U} \hat{K}_{Y} & \left(\hat{K}_{U}+\varepsilon I_{n}\right)^{2}
\end{array}\right) .
$$

We symmetrize the objective function by dividing by the constant $\operatorname{det} \hat{\Sigma}_{Y Y}$, which yields the objective function

$$
\frac{\operatorname{det} \hat{\Sigma}_{[Y U][Y U]}}{\operatorname{det} \hat{\Sigma}_{Y Y} \operatorname{det} \hat{\Sigma}_{U U}}
$$

We refer to the problem of minimizing this function with respect to the choice of subspace $S$ as Kernel Dimensionality Reduction (KDR).

Equation (14) has been termed the "kernel generalized variance" by Bach and Jordan (2002), who used it as a contrast function for independent component analysis. In that setting, the goal is to minimize a mutual information (among a set of recovered "source" variables), in the attempt to obtain independent components. Bach and Jordan (2002) showed that the kernel generalized variance is in fact an approximation of the mutual information of the recovered sources, when this mutual information is expanded around the manifold of factorized distributions. In the current setting, on the other hand, our goal is to maximize the mutual information $I(Y, U)$, and we certainly do not expect to be near a manifold in which $Y$ and $U$ are independent. Thus the argument for the kernel generalized variance as an objective function in the ICA setting does not apply here. What we have provided in the previous section is an entirely distinct argument that shows that the kernel generalized variance is in fact an appropriate objective function for the dimensionality reduction problem, and that minimizing the kernel generalized variance in Equation (14) can be viewed as a surrogate for maximizing the mutual information $I(Y, U)$, while the value of $I(Y, U)$ may not be explicitly related to the value of the kernel generalized variance.

For the optimization of Equation (14), we use gradient descent with line search in our experiments. In a straightforward implementation, the matrix $B$ is updated iteratively according to

$$
\begin{aligned}
B(t+1) & =B(t)-\eta \frac{\partial \log \operatorname{det} \hat{\Sigma}_{Y Y \mid U}}{\partial B} \\
& =B(t)-\eta \operatorname{Tr}\left[\hat{\Sigma}_{Y Y \mid U}^{-1} \frac{\partial \hat{\Sigma}_{Y Y \mid U}}{\partial B}\right],
\end{aligned}
$$

where $\eta$ is optimized through golden section search. The trace term in Equation (15) is rewritten by

$$
\operatorname{Tr}\left[\hat{\Sigma}_{Y Y \mid U}^{-1} \frac{\partial \hat{\Sigma}_{Y Y \mid U}}{\partial B}\right]=2 \varepsilon \operatorname{Tr}\left[\hat{\Sigma}_{Y Y \mid U}^{-1} \hat{K}_{Y}\left(\hat{K}_{U}+\varepsilon I_{n}\right)^{-1} \frac{\partial \hat{K}_{U}}{\partial B}\left(\hat{K}_{U}+\varepsilon I_{n}\right)^{-2} \hat{K}_{U} \hat{K}_{Y}\right] .
$$


All of these matrices are calculated directly from the definitions in Equations (12) and (13).

Given that the numerical task that must be solved in KDR is the same as the numerical task that must be solved in kernel ICA, we can import all of the computational techniques developed by Bach and Jordan (2002) for minimizing kernel generalized variance in the KDR setting. In particular, we can exploit incomplete Cholesky decomposition, which approximates an $n \times n$ positive semidefinite matrix $K$ by $K \approx A A^{T}$, where $A$ is an $n \times \ell$ matrix for $\ell<n$. Application of this decomposition reduces the $n \times n$ matrices $\hat{K}_{Y}$ and $\hat{K}_{U}$ required in Equation (15) to low-rank approximations. This diminishes the computational cost associated with multiplying and inverting large matrices, especially for a large $n$. For an exposition of incomplete Cholesky decomposition, see Bach and Jordan (2002). Another computational issue, which may arise in minimizing Equation (14), is the problem of local minima, because the objective function is not a convex function. To cope with this problem, we make use of an annealing technique, in which the scale parameter $\sigma$ for the Gaussian kernel is decreased gradually during the iterations of optimization. For a larger $\sigma$, the contrast function is smoother with fewer local optima, which makes optimization easier. The search becomes more accurate as $\sigma$ is decreased.

\section{Experimental Results}

We study the effectiveness of the new method through experiments, comparing it with several conventional methods: SIR, pHd, CCA, and PLS. For the experiments with SIR and pHd, we use an implementation in R due to Weisberg (2002).

In all of our experiments, we use a fixed value 0.1 for the regularization coefficient $\varepsilon$; empirically, the performance of the algorithm is robust to small variations in this coefficient. This coefficient could also be chosen using cross-validation.

\subsection{Synthetic Data}

The data sets Data I and Data II comprise one-dimensional $Y$ and two-dimensional $X=$ $\left(X_{1}, X_{2}\right)$. One hundred i.i.d. data points are generated by

$$
\begin{aligned}
\text { I : } & Y \sim 1 /\left(1+\exp \left(-X_{1}\right)\right)+Z, \\
\text { II }: & Y \sim 2 \exp \left(-X_{1}^{2}\right)+Z,
\end{aligned}
$$

where $Z \sim N\left(0,0.1^{2}\right)$, and $X=\left(X_{1}, X_{2}\right)$ follows a normal distribution and a normal mixture with two components for Data I and Data II, respectively. The effective subspace is spanned by $B_{0}=(1,0)^{T}$ in both cases. The data sets are depicted in Figure 2 .

Table 1 shows the angles between $B_{0}$ and the estimated direction. For Data I, all the methods except PLS yield a good estimate of $B_{0}$. Data II is surprisingly difficult for the conventional methods, presumably because the distribution of $X$ is not spherical and the regressor has a strong nonlinearity. This indicates the weakness of model-based methods; if data do not fit the model, the obtained result may not be meaningful. The KDR method succeeds in finding the correct direction for both data sets.

Data III has 300 samples of 17 dimensional $X$ and one dimensional $Y$, which are generated by

$$
\text { III }: \quad Y \sim 0.9 X_{1}+0.2 \frac{1}{1+X_{17}}+Z,
$$




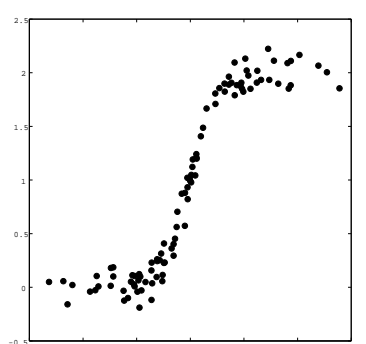

I: $\left(X_{1}, Y\right)$

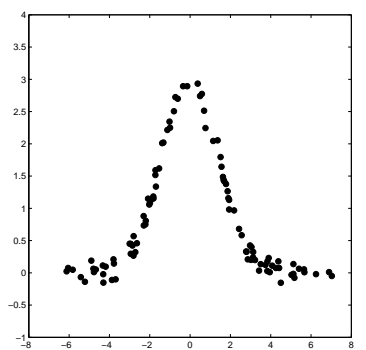

II: $\left(X_{1}, Y\right)$

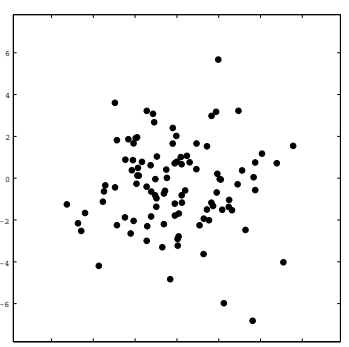

I: $\left(X_{1}, X_{2}\right)$

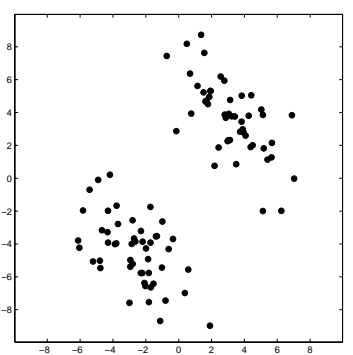

II: $\left(X_{1}, X_{2}\right)$

Figure 2: Data I and II. One-dimensional $Y$ depends only on $X_{1}$ in $X=\left(X_{1}, X_{2}\right)$.

\begin{tabular}{c||c|c|c|c|c}
\hline & SIR & pHd & CCA & PLS & Kernel \\
\hline I: angle (rad.) & 0.0087 & -0.1971 & 0.0099 & 0.2736 & -0.0014 \\
\hline II: angle (rad.) & -1.5101 & -0.9951 & -0.1818 & 0.4554 & 0.0052 \\
\hline
\end{tabular}

Table 1: Angles between the true and the estimated subspaces for Data I and II.

where $Z \sim N\left(0,0.01^{2}\right)$ and $X$ follows a uniform distribution on $[0,1]^{17}$. The effective subspace is given by $\boldsymbol{b}_{1}=(1,0, \ldots, 0)$ and $\boldsymbol{b}_{2}=(0, \ldots, 0,1)$. We compare the KDR method with SIR and pHd only - CCA and PLS cannot find a two-dimensional subspace, because $Y$ is one-dimensional. To evaluate the accuracy of the results, we use the multiple correlation coefficient

$$
R(\boldsymbol{b})=\max _{\boldsymbol{\beta} \in B} \frac{\boldsymbol{\beta}^{T} \Sigma_{X X} \boldsymbol{b}}{\sqrt{\boldsymbol{\beta}^{T} \Sigma_{X X} \boldsymbol{\beta} \cdot \boldsymbol{b}^{T} \Sigma_{X X} \boldsymbol{b}}}, \quad\left(\boldsymbol{b} \in B_{0}\right),
$$

which is used in Li (1991). As shown in Table 2, the KDR method outperforms the others in finding the weak contribution of the second direction.

\begin{tabular}{c||c|c|c|c|c|c}
\hline & $\operatorname{SIR}(10)$ & $\operatorname{SIR}(15)$ & $\operatorname{SIR}(20)$ & $\mathrm{SIR}(25)$ & $\mathrm{pHd}$ & Kernel \\
\hline$R\left(\boldsymbol{b}_{1}\right)$ & 0.987 & 0.993 & 0.988 & 0.990 & 0.110 & 0.999 \\
$R\left(\boldsymbol{b}_{2}\right)$ & 0.421 & 0.705 & 0.480 & 0.526 & 0.859 & 0.984 \\
\hline
\end{tabular}

Table 2: Correlation coefficients for Data III. SIR $(m)$ indicates SIR with $m$ slices. 


\begin{tabular}{c|c|c|c}
\hline Data set & dim. of $X$ & training sample & test sample \\
\hline Heart-disease & 13 & 149 & 148 \\
Ionosphere & 34 & 151 & 200 \\
Breast-cancer-Wisconsin & 30 & 200 & 369 \\
\hline
\end{tabular}

Table 3: Data description for the binary classification problem.

\subsection{Real Data: Classification}

In this section we apply the KDR method to classification problems. Many conventional methods of dimensionality reduction for regression are not suitable for classification. In particular, in the case of SIR, the dimensionality of the effective subspace must be less than the number of classes, because SIR uses the average of $X$ in slices along the variable $Y$. Thus, in binary classification, only a one-dimensional subspace can be found, because at most two slices are available. The methods CCA and PLS have a similar limitation on the dimensionality of the effective subspace; they cannot find a subspace of larger dimensionality than that of $Y$. Thus our focus is the comparison between KDR and pHd, which is applicable to general binary classification problems. Note that Cook and Lee (1999) discuss dimensionality reduction methods for binary classification, and propose the difference of covariance (DOC) method. They compare $\mathrm{pHd}$ and DOC theoretically, and show that these methods are the same in binary classification if the population ratio of the classes is $1 / 2$, which is almost the case in our experiments.

In the first experiment, we demonstrate the visualization capability of the dimensionality reduction methods. We use the Wine data set in the UCI machine learning repository (Murphy and Aha, 1994) to see how the projection onto a low-dimensional space realizes an effective description of data. The wine data consist of 178 samples with 13 variables and a label of three classes. We apply the KDR method, CCA, PLS, SIR, and pHd to these data. Figure 3 shows the projection onto the two-dimensional subspace estimated by each method. The KDR method separates the data into three classes most completely, while CCA also shows perfect separation. We can see that the data are nonlinearly separable in the two-dimensional space. The other methods do not separate the classes completely.

Next we investigate how much information on $Y$ is preserved in the estimated subspace. After reducing the dimensionality, we use the support vector machine (SVM) method to build a classifier in the reduced space, and compare its accuracy with an SVM trained using the full dimensional vector $X .^{2}$ Although we should theoretically use the (unknown) optimum classifier to evaluate the extent of the information preserved in the subspace, we use an SVM classifier whose parameters were chosen by exhaustive grid search. We use the Heart-disease data set, ${ }^{3}$ Ionosphere, and Wisconsin-breast-cancer from the UCI repository. A description of these data is presented in Table 3.

2. In our experiments with the SVM, we used the Matlab Support Vector Toolbox by S. Gunn; see http://www.isis.ecs.soton.ac.uk/resources/svminfo.

3. We use the Cleveland data set, created by Dr. Robert Detrano of V.A. Medical Center, Long Beach and Cleveland Clinic Foundation. Although the original data set has five classes, we use only "no presence" (0) and "presence" (1-4) for the binary class labels. Samples with missing values are removed in our experiments. 
Figure 4 shows the classification rates for the test set in subspaces of various dimensionality. We can see that KDR yields good separation even in low-dimensional subspaces, while pHd is much worse in low dimensions. It is noteworthy that in the Ionosphere data set the classifier in dimensions 5, 10, and 20 outperforms the classifier in the full dimensional space. This is presumably due to the suppression of noise irrelevant to the prediction of $Y$. These results show that the kernel method successfully finds a subspace which preserves the class information even when the dimensionality is reduced significantly.

\section{Extension to Variable Selection}

In this section, we describe an extension of the KDR method to the problem of variable selection. Variable selection is different from dimensionality reduction; the former involves selecting a subset of the explanatory variables $\left\{X_{1}, \ldots, X_{m}\right\}$ in order to obtain a simplified prediction of $Y$ from $X$, while the latter involves finding linear combinations of the variables. However, the objective function that we have presented for dimensionality reduction can be extended straightforwardly to variable selection. In particular, given a fixed number of variables to be selected, we can compare the KGV for subspaces spanned by combinations of this number of selected variables. This gives a reasonable way to select variables, because for a subset $W=\left\{X_{j_{1}}, \ldots, X_{j_{r}}\right\} \subset\left\{X_{1}, \ldots, X_{m}\right\}$, the variables $Y$ and $W^{C}$ are conditionally independent given $W$ if and only if $Y$ and $\Pi_{W^{c}} X$ are conditionally independent given $\Pi_{W} X$, where $\Pi_{W}$ and $\Pi_{W^{C}}$ are the orthogonal projections onto the subspaces spanned by $W$ and $W^{C}$, respectively. If we try to select $r$ variables from among $m$ explanatory variables, the total number of evaluations is $\left(\begin{array}{c}m \\ r\end{array}\right)$.

When $\left(\begin{array}{c}m \\ r\end{array}\right)$ is large, we must address the computational cost that arises in comparing large numbers of subsets. As in most other approaches to variable selection (see, e.g., Guyon and Elisseeff, 2003), we propose the use of a greedy algorithm and random search for this combinatorial aspect of the problem. (In the experiments presented in the current paper, however, we confine ourselves to small problems in which all combinations are tractably evaluated).

We apply this kernel-based method of variable selection to the Boston Housing data (Harrison and Rubinfeld, 1978) and the Ozone data (Breiman and Friedman, 1985), which have been often used as testbed examples for variable selection. Tables 4 and 5 give the detailed description of the data sets. There are 506 samples in the Boston Housing data, for which the variable MV, the median value of house prices in a tract, is estimated by using the 13 other variables. We use the corrected version of the data set given by Gilley and Pace (1996). In the Ozone data in which there are 330 samples, the variable UPO3 (the ozone concentration) is to be predicted by 9 other variables.

Table 6 shows the best three sets of four variables that attain the smallest values of the kernel generalized variance. For the Boston Housing data, RM and LSTAT are included in all the three of the result sets in Table 6, and PTRATIO and TAX are included in two of them. This observation agrees well with the analysis using alternating conditional expectation (ACE) by Breiman and Friedman (1985), which gives RM, LSTAT, PTRATIO, and TAX as the four major contributors. The original motivation in the study was to investigate the influence of nitrogen oxide concentration (NOX) on the house price (Harrison and Rubinfeld, 1978). In accordance with the previous studies, our analysis shows a rel- 


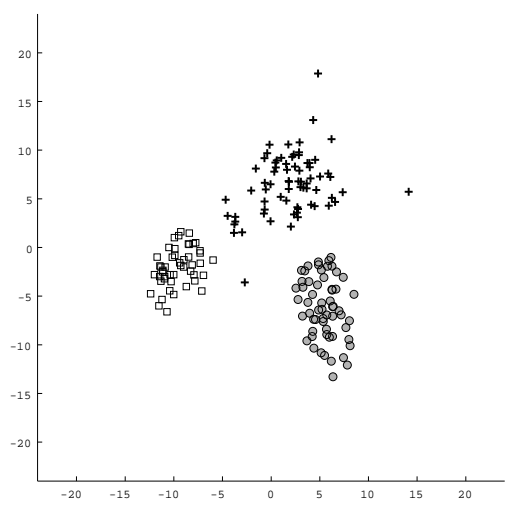

(a) KDR

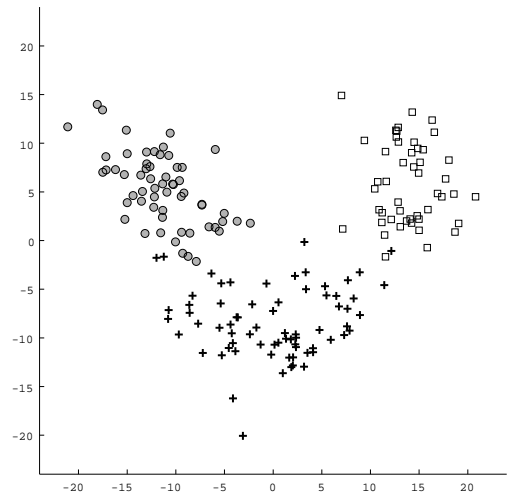

(c) PLS

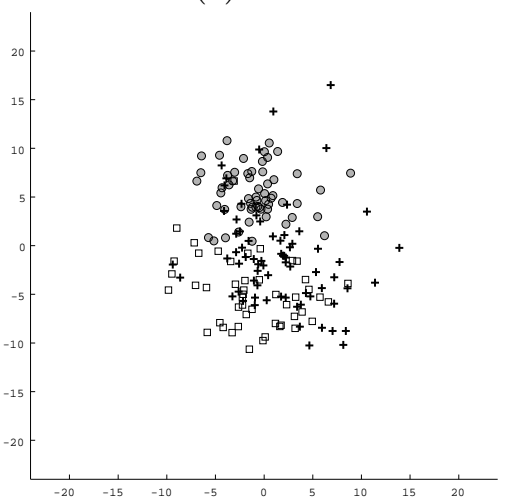

(e) $\mathrm{pHd}$

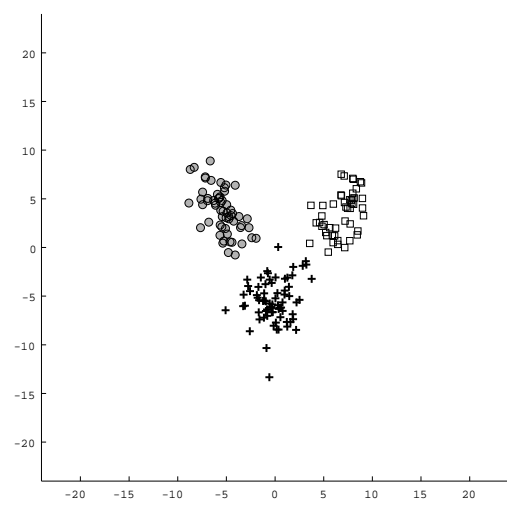

(b) CCA

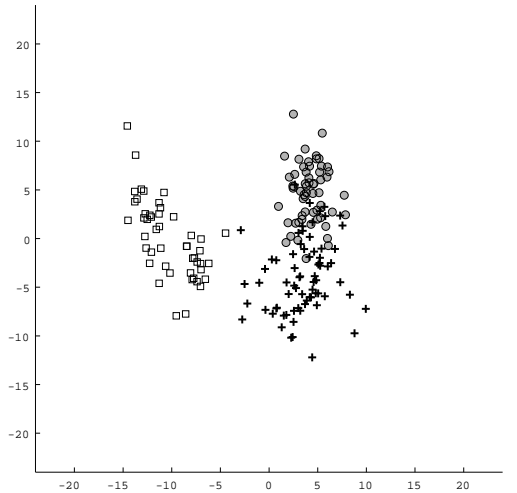

(d) SIR

Figure 3: Wine data. Projections onto the estimated two-dimensional space. The symbols ' + ', ' $\square$ ', and gray ' $\bigcirc$ ' represent the three classes. 
(a) Heart-disease

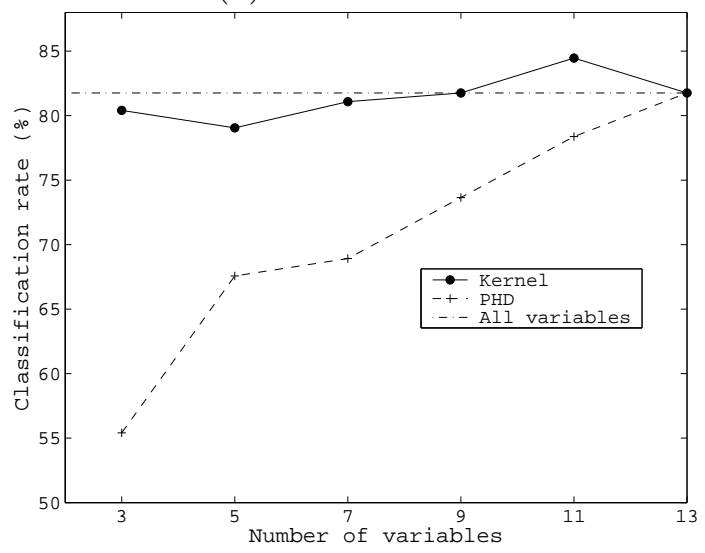

(b) Ionosphere

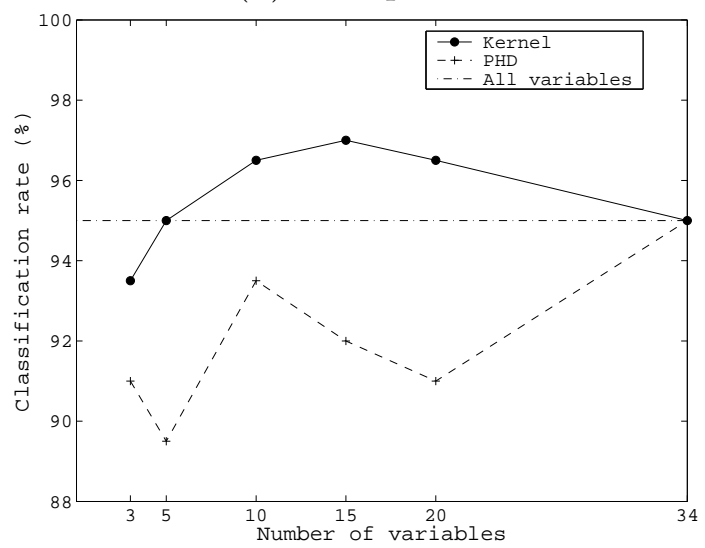

(c) Wisconsin Breast Cancer

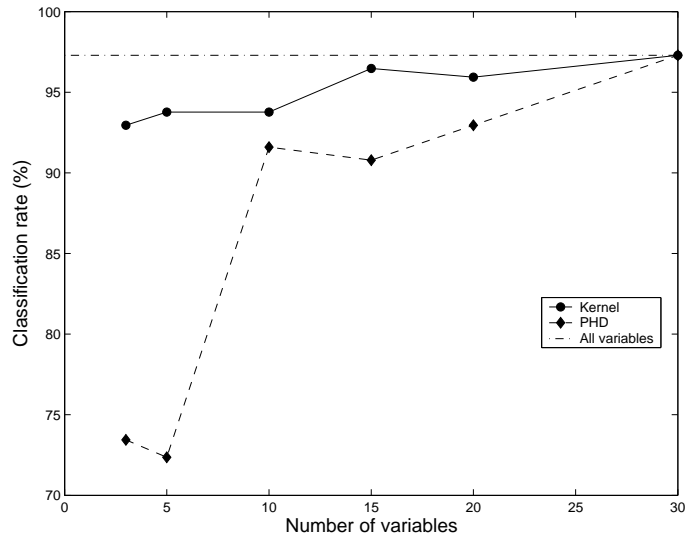

Figure 4: Classification accuracy of the SVM for test data after dimensionality reduction. 


\begin{tabular}{|c|c|c|}
\hline Variable & & Description \\
\hline MV & - & median value of owner-occupied home \\
\hline CRIM & - & crime rate by town \\
\hline $\mathrm{ZN}$ & - & $\begin{array}{l}\text { proportion of town's residential land zoned for lots } \\
\text { greater than } 25,000 \text { square feet }\end{array}$ \\
\hline INDUS & - & proportion of nonretail business acres per town \\
\hline CHAS & - & $\begin{array}{l}\text { Charles River dummy } \\
\qquad(=1 \text { if tract bounds the Charles River, } 0 \text { otherwise })\end{array}$ \\
\hline NOX & - & nitrogen oxide concentration in pphm \\
\hline $\mathrm{RM}$ & - & average number of rooms in owner units \\
\hline AGE & - & proportion of owner units build prior to 1940 \\
\hline DIS & - & $\begin{array}{l}\text { weighted distances to five employment centers } \\
\text { in the Boston region }\end{array}$ \\
\hline RAD & - & index of accessibility to radial highways \\
\hline TAX & - & full property tax rate $(\$ / \$ 10,000)$ \\
\hline PTRATIO & - & pupil-teacher ratio by town school district \\
\hline B & - & black proportion of population \\
\hline LSTAT & - & proportion of population that is lower status \\
\hline
\end{tabular}

Table 4: Boston Housing Data

\begin{tabular}{cll}
\hline Variable & Description \\
\hline UPO3 & - & upland ozone concentration $(\mathrm{ppm})$ \\
VDHT & - & Vandenburg 500 millibar height $(\mathrm{m})$ \\
HMDT & - & himidity (percent) \\
IBHT & - & inversion base height $(\mathrm{ft})$. \\
DGPG & - & Daggett pressure gradient $(\mathrm{mmhg})$ \\
IBTP & - & inversion base temperature $\left({ }^{\circ} \mathrm{F}\right)$ \\
SBTP & - & Sandburg Air Force Base temperature $\left({ }^{\circ} \mathrm{C}\right)$ \\
VSTY & - & visibility (miles) \\
WDSP & - & wind speed $(\mathrm{mph})$ \\
DAY & - & day of the year \\
\hline
\end{tabular}

Table 5: Ozone data

atively small contribution of NOX. For the Ozone data, all three of the result sets in the variable selection method include HMDT, SBTP, and IBHT. The variables IBTP, DGPG, and VDHT are chosen in one of the sets. This shows a fair accordance with earlier results by Breiman and Friedman (1985) and Li et al. (2000); the former concludes by ACE that SBTP, IBHT, DGPG, and VSTY are the most influential, and the latter selects HMDT, IBHT, and DGPG using a pHd-based method.

\section{Conclusion}

We have presented KDR, a new kernel-based approach to dimensionality reduction for regression and classification. One of the most notable aspects of this method is its generality- 


\begin{tabular}{|c|c|c|c|c|c|c|c|}
\hline Boston & $1 \mathrm{st}$ & 2nd & $3 \mathrm{rd}$ & & & & \\
\hline $\begin{array}{c}\text { CRIM } \\
\text { ZN } \\
\text { INDUS }\end{array}$ & \multirow{7}{*}{$\mathrm{X}$} & \multirow[t]{3}{*}{$\mathrm{X}$} & \multirow{5}{*}{$\mathrm{X}$} & & & & \\
\hline CHAS & & & & Ozone & 1st & 2nd & 3rd \\
\hline NOX & & & & VDHT & & & $\mathrm{X}$ \\
\hline $\mathrm{RM}$ & & \multirow[t]{4}{*}{$\mathrm{X}$} & & HMDT & $\mathrm{X}$ & $\mathrm{X}$ & $\mathrm{X}$ \\
\hline AGE & & & & IBHT & $\mathrm{X}$ & $\mathrm{X}$ & $\mathrm{X}$ \\
\hline DIS & & & \multirow[t]{2}{*}{$\mathrm{X}$} & DGPG & & $\mathrm{X}$ & \\
\hline RAD & & & & IBTP & $\mathrm{X}$ & & \\
\hline TAX & $\mathrm{X}$ & & $\mathrm{X}$ & SBTP & $\mathrm{X}$ & $\mathrm{X}$ & $\mathrm{X}$ \\
\hline PTRATIO & $\mathrm{X}$ & $\mathrm{X}$ & & VSTY & & & \\
\hline B & & & & WDSP & & & \\
\hline LSTAT & $\mathrm{X}$ & $\mathrm{X}$ & $\mathrm{X}$ & DAY & & & \\
\hline KGV & .1768 & .1770 & .1815 & KGV & .2727 & .2736 & .2758 \\
\hline
\end{tabular}

Table 6: Variable selection using the proposed kernel method.

we do not impose any strong assumptions on either the conditional or the marginal distribution. This allows the method to be applicable to a wide range of problems, and gives it a significant practical advantage over existing methods such as CCA, PPR, SIR, and $\mathrm{pHd}$. These methods all impose significant restrictions on the conditional probability, the marginal distribution, or the dimensionality of the effective subspaces.

Our experiments have shown that the KDR method can provide many of the desired effects of dimensionality reduction: it provides data visualization capabilities, it can successfully select important explanatory variables in regression, and it can yield classification performance that is better than the performance achieved with the full-dimensional covariate space. We have also discussed the extension of the KDR method to variable selection. Experiments with classical data sets has shown an accordance with the previous results on these data sets and suggest that further study of this application of KDR is warranted.

The theoretical basis of KDR lies in the nonparametric characterization of conditional independence that we have presented in this paper. Extending earlier work on the kernelbased characterization of independence in ICA (Bach and Jordan, 2002), we have shown that conditional independence can be characterized in terms of covariance operators on a reproducing kernel Hilbert space. While our focus has been on the problem of dimensionality reduction, it is also worth noting that there are many other possible applications of this characterization. In particular, conditional independence plays an important role in the structural definition of probabilistic graphical models, and our results may have applications to model selection and inference in graphical models.

There are several statistical problems which need to be addressed in further research on KDR. First, a basic analysis of the statistical consistency of the KDR-based estimatorthe convergence of the estimator to the true subspace when such a space really exists - is needed. We expect that, to prove consistency, we will need to impose a condition on the rate of decrease of the regularization coefficient $\varepsilon$ as the sample size $n$ goes to infinity. Second, and most significantly, we need rigorous methods for choosing the dimensionality 
of the effective subspace. If the goal is that of achieving high predictive performance after dimensionality reduction, we can use one of many existing methods (e.g., cross-validation, penalty-based methods) to assess the expected generalization as a function of dimensionality. Note in particular that by using KDR as a method to select an estimator given a fixed dimensionality, we have substantially reduced the number of hypotheses being considered, and expect to find ourselves in a regime in which methods such as cross-validation are likely to be effective. It is also worth noting, however, that the goals of dimensionality reduction are not always simply that of prediction; in particular, the search for small sets of explanatory variables will need to be guided by other principles. Finally, asymptotic analysis may provide useful guidance for selecting the dimensionality; an example of such an analysis that we believe can be adopted for KDR has been presented by Li (1991) for the SIR method.

\section{Acknowledgments}

This work was done while the first author was visiting the University of California, Berkeley. We thank the action editor and reviewers for their valuable comments. In particular, the proof of Theorem 6 was suggested by one of the reviewers. We also thank Dr. Noboru Murata of Waseda University and Dr. Motoaki Kawanabe of Fraunhofer, FIRST for their helpful comments on the early version of this work. We wish to acknowledge support from JSPS KAKENHI 15700241, ONR MURI N00014-00-1-0637, NSF grant IIS-9988642, and a grant from Intel Corporation.

\section{Appendix A. Cross-Covariance Operators on Reproducing Kernel Hilbert Spaces and Independence of Random Variables}

In this appendix, we present additional background and detailed proofs for results relating cross-covariance operators to marginal and conditional independence between random variables.

\section{A.1 Cross-Covariance Operators}

While cross-covariance operators are generally defined for random variables on Banach spaces Vakhania et al. (1987), Baker (1973), they are more easily defined on reproducing kernel Hilbert spaces (RKHS). In this subsection, we summarize some of the basic mathematical facts used in Sections 3.1 and 3.3. While we discuss only real Hilbert spaces, extension to the complex case is straightforward.

Theorem 1 Let $\left(\Omega_{1}, \mathcal{B}_{1}\right)$ and $\left(\Omega_{2}, \mathcal{B}_{2}\right)$ be measurable spaces, and let $\left(\mathcal{H}_{1}, k_{1}\right)$ and $\left(\mathcal{H}_{2}, k_{2}\right)$ be reproducing kernel Hilbert spaces on $\Omega_{1}$ and $\Omega_{2}$, respectively, with $k_{1}$ and $k_{2}$ measurable. Suppose we have a random vector $(X, Y)$ on $\Omega_{1} \times \Omega_{2}$ such that $E_{X}\left[k_{1}(X, X)\right]$ and $E_{Y}\left[k_{2}(Y, Y)\right]$ are finite. Then, there exists a unique operator $\Sigma_{Y X}$ from $\mathcal{H}_{1}$ to $\mathcal{H}_{2}$ such that

$$
\left\langle g, \Sigma_{Y X} f\right\rangle_{\mathcal{H}_{2}}=E_{X Y}[f(X) g(Y)]-E_{X}[f(X)] E_{Y}[g(Y)]
$$

holds for all $f \in \mathcal{H}_{1}$ and $g \in \mathcal{H}_{2}$. This is called the cross-covariance operator. 
Proof Obviously, the operator is unique, if it exists. From Riesz's representation theorem (see Reed and Simon, 1980, Theorem II.4, for example), the existence of $\Sigma_{Y X} f \in \mathcal{H}_{2}$ for a fixed $f$ can be proved by showing that the right hand side of Equation (16) is a bounded linear functional on $\mathcal{H}_{2}$. The linearity is obvious, and the boundedness is shown by

$$
\begin{aligned}
& \left|E_{X Y}[f(X) g(Y)]-E_{X}[f(X)] E_{Y}[g(Y)]\right| \\
& \leq E_{X Y}\left|\left\langle k_{1}(\cdot, X), f\right\rangle_{\mathcal{H}_{1}}\left\langle k_{2}(\cdot, Y), g\right\rangle_{\mathcal{H}_{2}}\right|+E_{X}\left|\left\langle k_{1}(\cdot, X), f\right\rangle_{\mathcal{H}_{1}}\right| \cdot E_{Y}\left|\left\langle k_{2}(\cdot, Y), g\right\rangle_{\mathcal{H}_{2}}\right| \\
& \leq E_{X Y}\left[\left\|k_{1}(\cdot, X)\right\|_{\mathcal{H}_{1}}\|f\|_{\mathcal{H}_{1}}\left\|k_{2}(\cdot, Y)\right\|_{\mathcal{H}_{2}}\|g\|_{\mathcal{H}_{2}}\right] \\
& \quad+E_{X}\left[\left\|k_{1}(\cdot, X)\right\|_{\mathcal{H}_{1}}\|f\|_{\mathcal{H}_{1}}\right] E_{Y}\left[\left\|k_{2}(\cdot, Y)\right\|_{\mathcal{H}_{2}}\|g\|_{\mathcal{H}_{2}}\right] \\
& \leq\left\{E_{X}\left[k_{1}(X, X)\right]^{1 / 2} E_{Y}\left[k_{2}(Y, Y)\right]^{1 / 2}+E_{X}\left[k_{1}(X, X)^{1 / 2}\right] E_{Y}\left[k_{2}(Y, Y)^{1 / 2}\right]\right\}\|f\|_{\mathcal{H}_{1}}\|g\|_{\mathcal{H}_{2}} \\
& \leq 2 E_{X}\left[k_{1}(X, X)\right]^{1 / 2} E_{Y}\left[k_{2}(Y, Y)\right]^{1 / 2}\|f\|_{\mathcal{H}_{1}}\|g\|_{\mathcal{H}_{2}} .
\end{aligned}
$$

For the second last inequality, $\|k(\cdot, x)\|_{\mathcal{H}}^{2}=k(x, x)$ is used. The linearity of the map $\Sigma_{Y X}$ is given by the uniqueness part of Riesz's representation theorem.

From Equation (17), $\Sigma_{Y X}$ is bounded, and by definition, we see $\Sigma_{Y X}^{*}=\Sigma_{X Y}$, where $A^{*}$ denotes the adjoint of $A$. If the two RKHS are the same, the operator $\Sigma_{X X}$ is called the covariance operator. A covariance operator $\Sigma_{X X}$ is bounded, self-adjoint, and trace-class.

In an RKHS, conditional expectations can be expressed by cross-covariance operators, in a manner analogous to finite-dimensional Gaussian random variables.

Theorem 2 Let $\left(\mathcal{H}_{1}, k_{1}\right)$ and $\left(\mathcal{H}_{2}, k_{2}\right)$ be RKHS on measurable spaces $\Omega_{1}$ and $\Omega_{2}$, respectively, with $k_{1}$ and $k_{2}$ measurable, and $(X, Y)$ be a random vector on $\Omega_{1} \times \Omega_{2}$. Assume that $E_{X}\left[k_{1}(X, X)\right]$ and $E_{Y}\left[k_{2}(Y, Y)\right]$ are finite, and for all $g \in \mathcal{H}_{2}$ the conditional expectation $E_{Y \mid X}[g(Y) \mid X=\cdot]$ is an element of $\mathcal{H}_{1}$. Then, we have for all $g \in \mathcal{H}_{2}$

$$
\Sigma_{X X} E_{Y \mid X}[g(Y) \mid X=\cdot]=\Sigma_{X Y} g,
$$

where $\Sigma_{X X}$ and $\Sigma_{X Y}$ are the covariance and cross-covariance operator.

Proof For any $f \in \mathcal{H}_{1}$, we have

$$
\begin{aligned}
& \left\langle f, \Sigma_{X X} E_{Y \mid X}[g(Y) \mid X=\cdot]\right\rangle_{\mathcal{H}_{1}} \\
& \quad=E_{X}\left[f(X) E_{Y \mid X}[g(Y) \mid X]\right]-E_{X}[f(X)] E_{X}\left[E_{Y \mid X}[g(Y) \mid X]\right] \\
& \quad=E_{X Y}[f(X) g(Y)]-E_{X}[f(X)] E_{Y}[g(Y)]=\left\langle f, \Sigma_{X Y} g\right\rangle_{\mathcal{H}_{1}} .
\end{aligned}
$$

This completes the proof.

Corollary 3 Let $\tilde{\Sigma}_{X X}^{-1}$ be the right inverse of $\Sigma_{X X}$ on $\left(\operatorname{Ker} \Sigma_{X X}\right)^{\perp}$. Under the same assumptions as Theorem 2, we have

$$
\left\langle f, \tilde{\Sigma}_{X X}^{-1} \Sigma_{X Y} g\right\rangle=\left\langle f, E_{Y \mid X}[g(Y) \mid X=\cdot]\right\rangle
$$

for all $f \in\left(\operatorname{Ker} \Sigma_{X X}\right)^{\perp}$ and $g \in \mathcal{H}_{2}$. In particular, if $\operatorname{Ker} \Sigma_{X X}=0$, we have

$$
\Sigma_{X X}^{-1} \Sigma_{X Y} g=E_{Y \mid X}[g(Y) \mid X=\cdot] .
$$


Proof Note that the product $\tilde{\Sigma}_{X X}^{-1} \Sigma_{X Y}$ is well-defined, because $\overline{\text { Range } \Sigma_{X Y}} \subset \overline{\text { Range } \Sigma_{X X}}=$ $\left(\operatorname{Ker} \Sigma_{X X}\right)^{\perp}$. The first inclusion is shown from the expression $\Sigma_{X Y}=\Sigma_{X X}^{1 / 2} V \Sigma_{Y Y}^{1 / 2}$ with a bounded operator $V$ (Baker, 1973, Theorem 1), and the second equation holds for any self-adjoint operator. Take $f=\Sigma_{X X} h \in \operatorname{Range} \Sigma_{X X}$. Then, Theorem 2 yields

$$
\begin{aligned}
\left\langle f, \tilde{\Sigma}_{X X}^{-1} \Sigma_{X Y} g\right\rangle & =\left\langle h, \Sigma_{X X} \tilde{\Sigma}_{X X}^{-1} \Sigma_{X X} E_{Y \mid X}[g(Y) \mid X=\cdot]\right\rangle \\
& =\left\langle h, \Sigma_{X X} E_{Y \mid X}[g(Y) \mid X=\cdot]\right\rangle=\left\langle f, E_{Y \mid X}[g(Y) \mid X=\cdot]\right\rangle .
\end{aligned}
$$

This completes the proof.

The assumption $E_{Y \mid X}[g(Y) \mid X=\cdot] \in \mathcal{H}_{1}$ in Theorem 2 can be simplified so that it can be checked without reference to a specific $g$.

Proposition 4 Under the condition of Theorem 2, if there exists $C>0$ such that

$$
E_{Y \mid X}\left[k_{2}\left(y_{1}, Y\right) \mid X=x_{1}\right] E_{Y \mid X}\left[k_{2}\left(y_{2}, Y\right) \mid X=x_{2}\right] \leq C k_{1}\left(x_{1}, x_{2}\right) k_{2}\left(y_{1}, y_{2}\right)
$$

for all $x_{1}, x_{2} \in \Omega_{1}$ and $y_{1}, y_{2} \in \Omega_{2}$, then for all $g \in \mathcal{H}_{2}$ the conditional expectation $E_{Y \mid X}[g(Y) \mid X=\cdot]$ is an element of $\mathcal{H}_{1}$.

Proof See Theorem 2.3.13 in Alpay (2001).

From this proposition, it is obvious that $E_{Y \mid X}[g(Y) \mid X=\cdot] \in \mathcal{H}_{1}$ holds, if the range of $X$ and $Y$ are bounded.

For a function $f$ in an RKHS, the expectation of $f(X)$ can be formulated as the inner product of $f$ and a fixed element. Let $(\Omega, \mathcal{B})$ be a measurable space, and $(\mathcal{H}, k)$ be an RKHS on $\Omega$ with $k$ measurable. Note that for a random variable $X$ on $\Omega$, the linear functional $f \mapsto E_{X}[f(X)]$ is bounded if $E_{X}[k(X, X)]$ exists. By Riesz's theorem, there is $u \in \mathcal{H}$ such that $\langle u, f\rangle_{\mathcal{H}}=E_{X}[f(X)]$ for all $f \in \mathcal{H}$. If we define $E_{X}[k(\cdot, X)] \in \mathcal{H}$ by this element $u$, we formally obtain the equality

$$
\left\langle E_{X}[k(\cdot, X)], f\right\rangle_{\mathcal{H}}=E_{X}\left[\langle k(\cdot, X), f\rangle_{\mathcal{H}}\right]
$$

which looks like the interchangeability of the expectation by $X$ and the inner product. While the expectation $E_{X}[k(\cdot, X)]$ can be defined, in general, as an integral with respect to the distribution on $\mathcal{H}$ induced by $k(\cdot, X)$, the element $E_{X}[k(\cdot, X)]$ is formally obtained as above in a reproducing kernel Hilbert space.

\section{A.2 Conditional Covariance Operator and Conditional Independence}

We define the conditional (cross-)covariance operator, and derive its relation with the conditional covariance of random variables. Let $\left(\mathcal{H}_{1}, k_{1}\right),\left(\mathcal{H}_{2}, k_{2}\right)$, let $\left(\mathcal{H}_{3}, k_{3}\right)$ be RKHS on measurable spaces $\Omega_{1}, \Omega_{2}$, and $\Omega_{3}$, respectively, and let $(X, Y, Z)$ be a random vector on $\Omega_{1} \times \Omega_{2} \times \Omega_{3}$. The conditional cross-covariance operator of $(X, Y)$ given $Z$ is defined by

$$
\Sigma_{Y X \mid Z}:=\Sigma_{Y X}-\Sigma_{Y Z} \tilde{\Sigma}_{Z Z}^{-1} \Sigma_{Z X} .
$$

Because $\operatorname{Ker} \Sigma_{Z Z} \subset \operatorname{Ker} \Sigma_{Y Z}$ from the fact $\Sigma_{Y Z}=\Sigma_{Y Y}^{1 / 2} V \Sigma_{Z Z}^{1 / 2}$ for some bounded operator $V$ (Baker, 1973, Theorem 1), the operator $\Sigma_{Y Z} \Sigma_{Z Z}^{-1} \Sigma_{Y X}$ can be uniquely defined, even if 
$\Sigma_{Z Z}^{-1}$ is not unique. By abuse of notation, we write $\Sigma_{Y Z} \Sigma_{Z Z}^{-1} \Sigma_{Z X}$, when cross-covariance operators are discussed.

The conditional cross-covariance operator is related to the conditional covariance of the random variables.

Proposition 5 Let $\left(\mathcal{H}_{1}, k_{1}\right),\left(\mathcal{H}_{2}, k_{2}\right)$, and $\left(\mathcal{H}_{3}, k_{3}\right)$ be reproducing kernel Hilbert spaces on measurable spaces $\Omega_{1}, \Omega_{2}$, and $\Omega_{3}$, respectively, with $k_{i}$ measurable, and let $(X, Y, Z)$ be a measurable random vector on $\Omega_{1} \times \Omega_{2} \times \Omega_{3}$ such that $E_{X}\left[k_{1}(X, X)\right], E_{Y}\left[k_{2}(Y, Y)\right]$, and $E_{Z}\left[k_{3}(Z, Z)\right]$ are finite. It is assumed that $E_{X \mid Z}[f(X) \mid Z=\cdot]$ and $E_{Y \mid Z}[g(Y) \mid Z=\cdot]$ are elements of $\mathcal{H}_{3}$ for all $f \in \mathcal{H}_{1}$ and $g \in \mathcal{H}_{2}$. Then, for all $f \in \mathcal{H}_{1}$ and $g \in \mathcal{H}_{2}$, we have

$$
\begin{aligned}
\left\langle g, \Sigma_{Y X \mid Z} f\right\rangle_{\mathcal{H}_{2}} & =E_{X Y}[f(X) g(Y)]-E_{Z}\left[E_{X \mid Z}[f(X) \mid Z] E_{Y \mid Z}[g(Y) \mid Z]\right] \\
& =E_{Z}\left[\operatorname{Cov}_{X Y \mid Z}(f(X), g(Y) \mid Z)\right] .
\end{aligned}
$$

Proof From the decomposition $\Sigma_{Y Z}=\Sigma_{Y Y}^{1 / 2} V \Sigma_{Z Z}^{1 / 2}$, we have $\Sigma_{Z Y} g \in\left(\operatorname{Ker} \Sigma_{Z Z}\right)^{\perp}$. Then, by Corollary 3, we obtain

$$
\begin{array}{r}
\left\langle g, \Sigma_{Y Z} \tilde{\Sigma}_{Z Z}^{-1} \Sigma_{Z X} f\right\rangle=\left\langle\Sigma_{Z Y} g, \tilde{\Sigma}_{Z Z}^{-1} \Sigma_{Z X} f\right\rangle=\left\langle\Sigma_{Z Y} g, E_{X \mid Z}[f(X) \mid Z]\right\rangle \\
=E_{Y Z}\left[g(Y) E_{X \mid Z}[f(X) \mid Z]\right]-E_{X}[f(X)] E_{Y}[g(Y)] .
\end{array}
$$

From this equation, the theorem is proved by

$$
\begin{aligned}
\left\langle g, \Sigma_{Y X \mid Z} f\right\rangle= & E_{X Y}[f(X) g(Y)]-E_{X}[f(X)] E_{Y}[g(Y)] \\
& \quad-E_{Y Z}\left[g(Y) E_{X \mid Z}[f(X) \mid Z]\right]+E_{X}[f(X)] E_{Y}[g(Y)] \\
= & E_{X Y}[f(X) g(Y)]-E_{Z}\left[E_{X \mid Z}[f(X) \mid Z] E_{Y \mid Z}[g(Y) \mid Z]\right] .
\end{aligned}
$$

The following definition is needed to state our main theorem. Let $(\Omega, \mathcal{B})$ be a measurable space, let $(\mathcal{H}, k)$ be a RKHS over $\Omega$ with $k$ measurable and bounded, and let $\mathcal{S}$ be the set of all the probability measures on $(\Omega, \mathcal{B})$. The RKHS $\mathcal{H}$ is called probability-determining, if the map

$$
\mathcal{S} \ni P \quad \mapsto \quad\left(f \mapsto E_{X \sim P}[f(X)]\right) \in \mathcal{H}^{*}
$$

is one-to-one, where $\mathcal{H}^{*}$ is the dual space of $\mathcal{H}$. From Riesz's theorem, $\mathcal{H}$ is probabilitydetermining if and only if the map

$$
\mathcal{S} \ni P \quad \mapsto \quad E_{X \sim P}[k(\cdot, X)] \in \mathcal{H}
$$

is one-to-one. For Gaussian kernels, the following theorem can be proved by an argument similar to that used in the proof of Theorem 2 in Bach and Jordan (2002) and the uniqueness of the characteristic function. For completeness, we present another simple proof here.

Theorem 6 For an arbitrary $\sigma>0$, the reproducing kernel Hilbert space $\mathcal{H}$ with Gaussian kernel $k(x, y)=\exp \left(-\|x-y\|^{2} / \sigma^{2}\right)$ on $\mathbb{R}^{m}$ is probability-determining. 
Proof Suppose $P$ and $Q$ are different probabilities on $\mathbb{R}^{m}$ such that $E_{Z \sim P}[f(Z)]=$ $E_{Z \sim Q}[f(Z)]$ for all $f \in \mathcal{H}$. Let $y_{1}$ and $y_{2}$ be two different vectors in $\mathbb{R}^{m}$, and $Y$ be a random variable with probability $1 / 2$ for each of $Y=y_{1}$ and $Y=y_{2}$. Define a random variable $X$ so that the probability of $X$ given $Y=y_{1}$ and $Y=y_{2}$ are $P$ and $Q$, respectively. Noting that the marginal distribution of $X$ is $(P+Q) / 2$, we have for all $f, g \in \mathcal{H}$,

$$
\begin{aligned}
& E_{X, Y}[f(X) g(Y)]-E_{X}[f(X)] E_{Y}[g(Y)]=E_{Y}\left[E_{X \mid Y}[f(X) \mid Y] g(Y)\right]-E_{X}[f(X)] E_{Y}[g(Y)] \\
& =\frac{1}{2} g\left(y_{1}\right) E_{X \mid Y}\left[f(X) \mid Y=y_{1}\right]+\frac{1}{2} g\left(y_{2}\right) E_{X \mid Y}\left[f(X) \mid Y=y_{2}\right] \\
& \quad-\frac{1}{2}\left(E_{Z \sim P}[f(Z)]+E_{Z \sim Q}[f(Z)]\right) \frac{g\left(y_{1}\right)+g\left(y_{2}\right)}{2} \\
& =0 .
\end{aligned}
$$

From Theorem 2 in Bach and Jordan (2002), $X$ and $Y$ must be independent, which contradicts the construction of $X$ and $Y$.

Recall that for two RKHS $\mathcal{H}_{1}$ and $\mathcal{H}_{2}$ on $\Omega_{1}$ and $\Omega_{2}$, respectively, the direct product $\mathcal{H}_{1} \otimes \mathcal{H}_{2}$ is the RKHS on $\Omega_{1} \times \Omega_{2}$ with the positive definite kernel $k_{1} k_{2}$ (see Aronszajn, 1950). Note that if the two RKHS have Gaussian kernels, their direct product is also a RKHS with Gaussian kernel, and thus probability-determining. The relation between conditional independence and the conditional covariance operator is given by the following theorem:

Theorem 7 Let $\left(\mathcal{H}_{11}, k_{11}\right),\left(\mathcal{H}_{12}, k_{12}\right)$, and $\left(\mathcal{H}_{2}, k_{2}\right)$ be reproducing kernel Hilbert spaces on measurable spaces $\Omega_{11}, \Omega_{12}$, and $\Omega_{2}$, respectively, with continuous and bounded kernels. Let $(X, Y)=(U, V, Y)$ be a random vector on $\Omega_{11} \times \Omega_{12} \times \Omega_{2}$, where $X=(U, V)$, and let $\mathcal{H}_{1}=\mathcal{H}_{11} \otimes \mathcal{H}_{12}$ be the direct product. It is assumed that $E_{Y \mid U}[g(Y) \mid U=\cdot] \in \mathcal{H}_{11}$ and $E_{Y \mid X}[g(Y) \mid X=\cdot] \in \mathcal{H}_{1}$ for all $g \in \mathcal{H}_{2}$. Then, we have

$$
\Sigma_{Y Y \mid U} \geq \Sigma_{Y Y \mid X}
$$

where the inequality refers to the order of self-adjoint operators, and if further $\mathcal{H}_{2}$ is probability-determining, the following equivalence holds

$$
\Sigma_{Y Y \mid X}=\Sigma_{Y Y \mid U} \quad \Longleftrightarrow \quad Y \Perp V \mid U .
$$

Proof The right hand side of Equation (21) is equivalent to $P_{Y \mid X}=P_{Y \mid U}$, where $P_{Y \mid X}$ and $P_{Y \mid U}$ are the conditional probability of $Y$ given $X$ and given $U$, respectively. Taking the expectation of the well-known equality

$$
\operatorname{Var}_{Y \mid U}[g(Y) \mid U]=E_{V \mid U}\left[\operatorname{Var}_{Y \mid U, V}[g(Y) \mid U, V]\right]+\operatorname{Var}_{V \mid U}\left[E_{Y \mid U, V}[g(Y) \mid U, V]\right]
$$

with respect to $U$, we derive

$$
E_{U}\left[\operatorname{Var}_{Y \mid U}[g(Y) \mid U]\right]=E_{X}\left[\operatorname{Var}_{Y \mid X}[g(Y) \mid X]\right]+E_{U}\left[\operatorname{Var}_{V \mid U}\left[E_{Y \mid X}[g(Y) \mid X]\right]\right] .
$$

Since the last term of Equation (22) is nonnegative, we obtain Equation (20) from Proposition 5 . 
Equality holds if and only if $\operatorname{Var}_{V \mid U}\left[E_{Y \mid X}[g(Y) \mid X]\right]=0$ for almost every $U$, which means $E_{Y \mid X}[g(Y) \mid X]$ does not depend on $V$ almost surely. This is equivalent to

$$
E_{Y \mid X}[g(Y) \mid X]=E_{Y \mid U}[g(Y) \mid U]
$$

for almost every $V$ and $U$. Because $\mathcal{H}_{2}$ is probability-determining, this means $P_{Y \mid X}=P_{Y \mid U}$.

\section{A.3 Conditional Cross-Covariance Operator and Conditional Independence}

Theorem 7 characterizes conditional independence using the conditional covariance operator. Another formulation is possible with a conditional cross-covariance operator.

Let $\left(\Omega_{1}, \mathcal{B}_{1}\right),\left(\Omega_{2}, \mathcal{B}_{2}\right)$, and $\left(\Omega_{3}, \mathcal{B}_{3}\right)$ be measurable spaces, and let $(X, Y, Z)$ be a random vector on $\Omega_{1} \times \Omega_{2} \times \Omega_{3}$ with law $P_{X Y Z}$. We define a probability measure $E_{Z}\left[P_{X \mid Z} \otimes P_{Y \mid Z}\right]$ on $\Omega_{1} \times \Omega_{2}$ by

$$
E_{Z}\left[P_{X \mid Z} \otimes P_{Y \mid Z}\right](A \times B)=E_{Z}\left[E_{X \mid Z}\left[\chi_{A} \mid Z\right] E_{Y \mid Z}\left[\chi_{B} \mid Z\right]\right]
$$

where $\chi_{A}$ is the characteristic function of a measurable set $A$. It is canonically extended to any product-measurable sets in $\Omega_{1} \times \Omega_{2}$.

Theorem 8 Let $\left(\Omega_{i}, \mathcal{B}_{i}\right)(i=1,2,3)$ be a measurable space, let $\left(\mathcal{H}_{i}, k_{i}\right)$ be a $R K H S$ on $\Omega_{i}$ with kernel measurable and bounded, and let $(X, Y, Z)$ be a random vector on $\Omega_{1} \times \Omega_{2} \times \Omega_{3}$. It is assumed that $E_{X \mid Z}[f(X) \mid Z=\cdot]$ and $E_{Y \mid Z}[g(Y) \mid Z=\cdot]$ belong to $\mathcal{H}_{3}$ for all $f \in \mathcal{H}_{1}$ and $g \in \mathcal{H}_{2}$, and that $\mathcal{H}_{1} \otimes \mathcal{H}_{2}$ is probability-determining. Then, we have

$$
\Sigma_{Y X \mid Z}=O \quad \Longleftrightarrow \quad P_{X Y}=E_{Z}\left[P_{X \mid Z} \otimes P_{Y \mid Z}\right] .
$$

Proof The right-to-left direction is trivial from Theorem 5 and the definition of $E_{Z}\left[P_{X \mid Z} \otimes\right.$ $\left.P_{Y \mid Z}\right]$. The left-hand side yields $E_{Z}\left[E_{X \mid Z}[f(X) \mid Z] E_{Y \mid Z}[g(Y) \mid Z]\right]=E_{X Y}[f(X) g(Y)]$ for all $f \in \mathcal{H}_{1}$ and $g \in \mathcal{H}_{2}$. Because $\mathcal{H}_{1} \otimes \mathcal{H}_{2}$ is defined as the completion of all the linear combinations of $f_{i}(x) g_{i}(y)$ for $f_{i} \in \mathcal{H}_{1}$ and $g_{i} \in \mathcal{H}_{2}$, we have $E_{\left(X^{\prime}, Y^{\prime}\right) \sim Q}\left[h\left(X^{\prime}, Y^{\prime}\right)\right]=$ $E_{X Y}[h(X, Y)]$ for every such linear combination, and thus every $h \in \mathcal{H}_{1} \otimes \mathcal{H}_{2}$ as a limit, where $Q=E_{Z}\left[P_{X \mid Z} \otimes P_{Y \mid Z}\right]$. This implies the right-hand side, because $\mathcal{H}_{1} \otimes \mathcal{H}_{2}$ is probability-determining.

The right-hand side of Equation (23) is weaker than the conditional independence of $X$ and $Y$ given $Z$. However, if $Z$ is a part of $X$, we obtain conditional independence.

Corollary 9 Let $\left(\mathcal{H}_{11}, k_{11}\right),\left(\mathcal{H}_{12}, k_{12}\right)$, and $\left(\mathcal{H}_{2}, k_{2}\right)$ be reproducing kernel Hilbert spaces on measurable spaces $\Omega_{11}, \Omega_{12}$, and $\Omega_{2}$, respectively, with kernels measurable and bounded. Let $(X, Y)=(U, V, Y)$ be a random vector on $\Omega_{11} \times \Omega_{12} \times \Omega_{2}$, where $X=(U, V)$, and let $\mathcal{H}_{1}=\mathcal{H}_{11} \otimes \mathcal{H}_{12}$ be the direct product. It is assumed that $E_{X \mid U}[f(X) \mid U=\cdot]$ and $E_{Y \mid U}[g(Y) \mid U=\cdot]$ belong to $\mathcal{H}_{11}$ for all $f \in \mathcal{H}_{1}$ and $g \in \mathcal{H}_{2}$, and $\mathcal{H}_{1} \otimes \mathcal{H}_{2}$ is probabilitydetermining. Then, we have

$$
\Sigma_{Y X \mid U}=O \quad \Longleftrightarrow \quad Y \Perp V \mid U .
$$


Proof For any measurable sets $A \subset \Omega_{11}, B \subset \Omega_{12}$, and $C \subset \Omega_{2}$, we have, in general,

$$
\begin{aligned}
& E_{U}\left[E_{X \mid U}\left[\chi_{A \times B}(U, V) \mid U\right] E_{Y \mid U}\left[\chi_{C}(Y) \mid U\right]\right]-E_{X Y}\left[\chi_{A \times B}(U, V) \chi_{C}(Y)\right] \\
& =E_{U}\left[E_{V \mid U}\left[\chi_{B}(V) \mid U\right] \chi_{A}(U) E_{Y \mid U}\left[\chi_{C}(Y) \mid U\right]\right]-E_{U}\left[E_{V Y \mid U}\left[\chi_{B}(V) \chi_{C}(Y) \mid U\right] \chi_{A}(U)\right] \\
& =\int_{A}\left\{P_{V \mid U}(B \mid u) P_{Y \mid U}(C \mid u)-P_{V Y \mid U}(B \times C \mid u)\right\} d P_{U}(u) .
\end{aligned}
$$

From Theorem 8, the left-hand side of Equation (24) is equivalent to $E_{U}\left[P_{X \mid U} \otimes P_{Y \mid U}\right]=$ $P_{X Y}$, which implies that the last integral in Equation (25) is zero for all $A$. This means $P_{V \mid U}(B \mid u) P_{Y \mid U}(C \mid u)-P_{V Y \mid U}(B \times C \mid u)=0$ for almost every $u-P_{U}$. Thus, $Y$ and $V$ are conditional independent given $U$. The converse is trivial.

Note that the left-hand side of Equation (24) is not $\Sigma_{Y V \mid U}$ but $\Sigma_{Y X \mid U}$, which is defined on the direct product $\mathcal{H}_{11} \otimes \mathcal{H}_{12}$.

\section{References}

Daniel Alpay. The Schur Algorithm, Reproducing Kernel Spaces and System Theory. American Mathematical Society, 2001.

Nachman Aronszajn. Theory of reproducing kernels. Transactions of the American Mathematical Society, 69(3):337-404, 1950.

Francis R. Bach and Michael I. Jordan. Kernel independent component analysis. Journal of Machine Learning Research, 3:1-48, 2002.

Francis R. Bach and Michael I. Jordan. Beyond independent components: trees and clusters. Journal of Machine Learning Research, 2003a. In press.

Francis R. Bach and Michael I. Jordan. Learning graphical models with Mercer kernels. In S. Becker, S. Thrun, and K. Obermayer, editors, Advances in Neural Information Processing Systems 15. MIT Press, 2003b.

Charles R. Baker. Joint measures and cross-covariance operators. Transactions of the American Mathematical Society, 186:273-289, 1973.

Christopher M. Bishop. Neural Networks for Pattern Recognition. Oxford University Press, 1995.

Bernhard E. Boser, Isabelle M. Guyon, and Vladimir N. Vapnik. A training algorithm for optimal margin classifiers. In D. Haussler, editor, Fifth Annual ACM Workshop on Computational Learning Theory, pages 144-152. ACM Press, 1992.

Leo Breiman and Jerome H. Friedman. Estimating optimal transformations for multiple regression and correlation. Journal of the American Statistical Association, 80:580-598, 1985.

R. Dennis Cook. Regression Graphics. Wiley Inter-Science, 1998. 
R. Dennis Cook and Hakbae Lee. Dimension reduction in regression with a binary response. Journal of the American Statistical Association, 94:1187-1200, 1999.

R. Dennis Cook and S. Weisberg. Discussion of Li (1991). Journal of the American Statistical Association, 86:328-332, 1991.

R. Dennis Cook and Xiangrong Yin. Dimension reduction and visualization in discriminant analysis (with discussion). Australian $\& 3$ New Zealand Journal of Statistics, 43(2):147-199, 2001.

Jerome H. Friedman and Werner Stuetzle. Projection pursuit regression. Journal of the American Statistical Association, 76:817-823, 1981.

Wing Kam Fung, Xuming He, Li Liu, and Peide Shi. Dimension reduction based on canonical correlation. Statistica Sinica, 12(4):1093-1114, 2002.

Otis W. Gilley and R. Kelly Pace. On the Harrison and Rubingeld data. Journal of Environmental Economics Management, 31:403-405, 1996.

Isabelle Guyon and André Elisseeff. An introduction to variable and feature selection. Journal of Machine Learning Research, 3:1157-1182, 2003.

David Harrison and Daniel L. Rubinfeld. Hedonic housing prices and the demand for clean air. Journal of Environmental Economics Management, 5:81-102, 1978.

Trevor Hastie and Robert Tibshirani. Generalized additive models. Statistical Science, 1: 297-318, 1986.

Inge S. Helland. On the structure of partial least squares. Communications in Statistics Simulations and Computation, 17(2):581-607, 1988.

Agnar Höskuldsson. PLS regression methods. Journal of Chemometrics, 2:211-228, 1988.

Marian Hristache, Anatoli Juditsky, Jörg Polzehl, and Vladimir Spokoiny. Structure adaptive approach for dimension reduction. The Annals of Statistics, 29(6):1537-1566, 2001.

Ker-Chau Li. Sliced inverse regression for dimension reduction (with discussion). Journal of the American Statistical Association, 86:316-342, 1991.

Ker-Chau Li. On principal Hessian directions for data visualization and dimension reduction: Another application of Stein's lemma. Journal of the American Statistical Association, 87:1025-1039, 1992.

Ker-Chau Li, Heng-Hui Lue, and Chun-Houh Chen. Interactive tree-structured regression via principal Hessian directions. Journal of the American Statistical Association, 95(450): 547-560, 2000.

Patrick M. Murphy and David W. Aha. UCI repository of machine learning databases. Technical report, University of California, Irvine, Department of Information and Computer Science. http://www.ics.uci.edu/〜 mlearn/MLRepository.html, 1994. 
Radford M. Neal. Bayesian Learning for Neural Networks. Springer Verlag, 1996.

Danh V. Nguyen and David M. Rocke. Tumor classification by partial least squares using microarray gene expression data. Bioinformatics, 18(1):39-50, 2002.

Michael Reed and Barry Simon. Functional Analysis. Academic Press, 1980.

Alexander M. Samarov. Exploring regression structure using nonparametric functional estimation. Journal of the American Statistical Association, 88(423):836-847, 1993.

Bernhard Schölkopf, Alexander Smola, and Klaus-Robert Müller. Nonlinear component analysis as a kernel eigenvalue problem. Neural Computation, 10:1299-1319, 1998.

Kari Torkkola. Feature extraction by non-parametric mutual information maximization. Journal of Machine Learning Research, 3:1415-1438, 2003.

Nikolai N. Vakhania, Vazha I. Tarieladze, and Sergei A. Chobanyan. Probability Distributions on Banach Spaces. D. Reidel Publishing Company, 1987.

Vladimir N. Vapnik, Steven E. Golowich, and Alexander J. Smola. Support vector method for function approximation, regression estimation, and signal processing. In M. Mozer, M. Jordan, and T. Petsche, editors, Advances in Neural Information Processing Systems 9, pages 281-287. MIT Press, 1997.

Francesco Vivarelli and Christopher K.I. Williams. Discovering hidden features with Gaussian process regression. In Michael Kearns, Sara Solla, and David Cohn, editors, Advances in Neural Processing Systems, volume 11, pages 613-619. MIT Press, 1999.

Sanford Weisberg. Dimension reduction regression in R. Journal of Statistical Software, 7 (1), 2002. 